\title{
Book Review: Bätzing, Werner (2020): Das Landleben. Geschichte und Zukunft einer gefährdeten Lebensform \\ München: C. H. Beck. 302 Seiten, 27 überwiegend farbige Abbildungen, 3 Karten und 3 Tabellen.
}

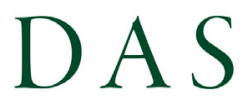

1

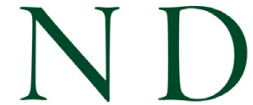

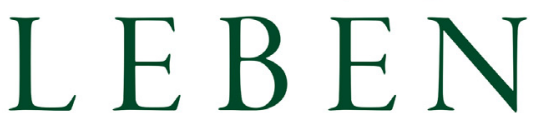

Geschichte und Zukunft einer gefährdeten Lebensform

\section{WERNER B ÄTZING} C.H.Beck

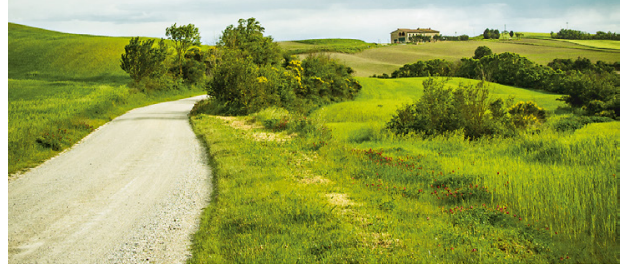

Werner Bätzing, der bisher vor allem durch sehr kenntnisreiche Bücher über den Alpenraum bekannt wurde, hat eine umfassende Analyse des Landlebens vorgelegt. Landleben setzt sich vom Stadtleben ab, daher muss im Buch auch immer wieder zwischen Land und Stadt verglichen werden. Der Autor stellt sein Thema, die Entwicklung des Lebens auf dem Land, in einem historischen Abriss vor.

Landleben gibt es auf der Erde seit der Zeit, in der Kulturpflanzen aus Wildpflanzen entwickelt wurden. Dabei veränderten sich nicht nur die Pflanzen, sondern auch die Menschen, die zu Bauern wurden und sesshaft leben mussten, um im Sommer ihre Felder zu bestellen und im Winter ihre Vorräte zu schützen. Dies setzte vor etwa 12.000 Jahren ein. Werner Bätzing geht von einer "ökologischen Reproduktion" aus, die von den frühen Bauern geleistet wurde. Sie bauten also nicht nur Kulturpflanzen an und hielten Haustiere, sondern: „Zentrale Frage der traditionellen Landwirtschaft ist stets die ökologische Reproduktion der Kulturlandschaft und nicht, ob Nutzungen arbeitsintensiv oder -extensiv ausgeführt werden" (S. 42). Dieser Gedanke ist allerdings Wunschdenken, denn ländliche Siedlungen blieben in prähistorischer Zeit nie länger als ein paar Jahrzehnte oder allenfalls Jahrhunderte bestehen.

Mit der Entstehung von Städten und Hochkulturen ist das Land, wie Werner Bätzing darstellt, schon frühzeitig entwertet worden. Im europäischen Mittelalter seien Land und Stadt gleichwertig gewesen, ein interessanter Gedanke, der dadurch an Schlagkraft gewinnen

*Corresponding author: Prof. Dr. Hansjörg Küster, Leibniz Universität Hannover, Institut für Geobotanik, Nienburger Straße 17, 30167 Hannover, Deutschland, E-Mail: kuester@geobotanik.uni-hannover.de 
würde, wenn davon die Rede wäre, dass damals auch ländliche Siedlungen ortsfest wurden und in aller Regel nicht mehr verlagert wurden. Dafür sorgte nicht nur die Anbindung der ländlichen Siedlung an eine (Wasser-) Mühle, die erwähnt wird, sondern auch die Gruppierung der Höfe um eine feste Kirche, von der nicht die Rede ist. Auch die im Flurzwang bewirtschafteten Langstreifenfluren, deren Anlage aufwendig war, verhinderten eine Verlagerung von Siedlungen und Fluren. Sie wurden später reformiert. Werner Bätzing beschreibt die Gemeinheitsteilungen, also die Aufteilung der Allmende unter den einzelnen Bauern, und die enclosures oder Hecken, mit denen die Flächen der ehemaligen Allmende untereinander abgeteilt wurden, aber nicht die damit in Verbindung stehende Aneinanderkopplung der Langstreifen zu einer Koppel, die ein großflächigeres und unabhängigeres Wirtschaften der einzelnen Bauern ermöglichte. Die alles beherrschende Industrielle Revolution läutete das "fossile Zeitalter" ein, in dem die Gewinnung fossiler Energieträger wichtiger wurde als nachwachsende Rohstoffe, die zur gleichen Zeit entstanden, in der man sie nutzte, gemeint ist vor allem Holz. Es dominierte nun wieder die Stadt: Sie war attraktiv und bot Aufstiegsmöglichkeiten. Das Land hingegen fiel zurück und wurde vom wirtschaftlichen Fortschritt abgekoppelt.

Es folgen die interessantesten Teile des Buches, in denen der Autor die Entwicklungen auf dem Land klar zueinander in Beziehung setzt. Die Tradition erhielt eine neue „Aufgabe und einen neuen Sinn, nämlich die Bewältigung des schnellen gesellschaftlichen Wandels" (S. 112). Zugleich wurden seit der zweiten Hälfte des 19. Jahrhunderts die „richtigen“ Traditionen oft erst erfunden; sie wurden eingeführt und galten vielfach schon nach wenigen Jahren als "uralt“. Aus der Sicht der Städter wurde auch das Land als "schöne Landschaft" neu wahrgenommen.

Zwischen 1960 und 1980 kam es, wie Werner Bätzing weiter ausführt, zu einer forcierten Modernisierung des Landlebens, etwa durch die Massenmotorisierung. Zugleich wurden die Bauern zur Minderheit im Dorf, die Bewirtschaftung des Landes war aber nicht nur durch Intensivierung, sondern auch durch Extensivierung geprägt. Große Schulzentren wurden eingeführt, was interessante Nebeneffekte hatte: Die Lehrer wohnten nicht mehr unbedingt im gleichen Ort wie die Schüler, der Unterricht wurde immer weniger auf die Verhältnisse am Wohnort der Schüler bezogen. Dafür wurde er verwissenschaftlicht und vereinheitlicht.

Werner Bätzing zeichnet nach, wie die Raumordnung immer stärker in die Entwicklung eingriff. Mit der Gemeindereform verloren viele Dörfer ihre Selbststän- digkeit. Man definierte Oberzentren, in denen allein alle Funktionen eines städtischen Zentrums gewährleistet sein sollten. Von überall auf dem Land sollten diese Dienstleistungszentren in einer Stunde erreichbar sein, wofür Autobahnen und Bundesstraßen gebaut wurden. Das Landleben galt nun als Auslaufmodell, die Dorfkerne wurden verlassen, Neubauten entstanden, wenn überhaupt, dann am Dorfrand. Und nur wenige Jahre später waren die in den 1960er- und 1970er-Jahren geschaffenen Strukturen überholt. Denn in einer späteren ,postmodernen' Phase wurde das Landleben einerseits aufgewertet, auf der anderen Seite mag es völlig verschwunden sein. Das Land wurde zwar neu entdeckt und die Dorfsanierung wandelte sich zur Dorferneuerung. Interessant ist aber, dass das typische dörfliche Miteinander mit der gegenseitigen (unbezahlten) Hilfe verschwand und stattdessen bezahlte Dienstleistungen eingeführt wurden.

Einerseits kamen, so Werner Bätzing weiter, immer mehr Großbetriebe der Landwirtschaft auf, andererseits die ökologische Landwirtschaft und die Produktion von Regionalprodukten. Es gab immer weniger Dienstleister auf dem Land (beispielsweise Lebensmittelläden), eine Entwicklung, die dann aufgehalten werden konnte, wenn sich ländliche Industrie auf dem Land ansiedeln konnte. Tatsächlich entwickelten sich viele kleinere Industriebetriebe zu sogenannten hidden champions. Doch wie wird es weitergehen? „Das Bild eines homogenen ländlichen Raumes macht aus dem Landleben ein Idyll, ein realitätsfernes Zerrbild, das erstmals gegen Ende des 19. Jahrhunderts als Anti-Position zur dynamischen Industriestadt im Rahmen des Heimatschutzes entsteht [...]. Als politische Leitidee löst es aber kein einziges der aktuellen Probleme, und als falsches Versprechen führt es letztlich in den Abgrund" (S. 210). Und der Autor kommt zum Fazit: „Ich gehe davon aus, dass ländlicher Raum und Landleben seit Beginn der Industriellen Revolution zwar deutlich geschwächt und entwertet wurden, dass aber beide weiterhin existieren und dass ihr Verschwinden keineswegs kurz bevorsteht" (S. 215). Man muss sich, so Werner Bätzing, über die Gefahren, denen ländliches Leben ausgesetzt ist, allerdings auch im Klaren sein: Ein totaler Neoliberalismus würde den ländlichen Raum zusammenbrechen lassen. Eine globale Wirtschaftskrise und hohe Benzinpreise würden das Land schwer treffen, schädlich ist aber auch die Entstehung von Wildnissen auf dem Land, weil sie bäuerliches Kulturland verschwinden lässt.

Werner Bätzing regt dazu an, die kulturelle Identität des ländlichen Raumes als Schlüsselfaktor zu sehen. Man sollte stärker darauf achten, lokal Typisches zu 
produzieren. Und ländliche Dienstleistungen, die nur in geringem Umfang geleistet werden müssen, setzen voraus, dass die Dienstleister mehreren verschiedenen Tätigkeiten nachgehen. Da die Steuergesetze nur von einer oder allenfalls zwei Tätigkeiten ausgehen, sind hier Innovationen bei der steuerlichen Behandlung erforderlich. Ländliche Infrastrukturen müssen gestärkt, Verkehrsmittel besser vernetzt werden, das Landleben bräuchte neue Raumstrukturen, beispielsweise über Landkreisgrenzen hinweg. Im Ganzen könnte ein neuer Blick auf das Landleben, so Bätzing, „zur Grundlage dafür werden, dass man sich nicht mehr den Sachzwängen einer unüberschaubar gewordenen Welt ausgeliefert fühlt, sondern selbst aktiv wird" (S. 255).

Werner Bätzing fügt in seinem Buch die Fakten gelegentlich etwas holzschnittartig aneinander und verallgemeinert zu stark. Das kann man kritisieren, aber auch als Vorteil sehen, weil dadurch die Vernetzung zwischen den einzelnen Sachverhalten umso deutlicher zu Tage tritt. Das Buch bietet daher eine Bestandsaufnahme ländlichen Lebens in der Gegenwart, allerdings in einer Zeit, in der es unerwartet zu einem tiefgreifenden Umbruch unseres täglichen Lebens gekommen ist: Die CoronaKrise, die ganz kurz nach dem Erscheinen des Buches einsetzte, könnte erhebliche Veränderungen gerade in der Wertschätzung des Landes mit sich bringen, die in ihren Auswirkungen noch kaum zu erahnen sind. Dass nur das ,Selbst-aktiv-Werden' auf dem Land weiterführt, gilt aber für die Bewältigung der Krise in noch viel höherem Maße. 